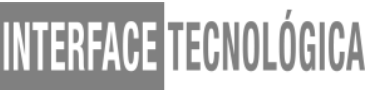

\section{AS REVOLUÇÕES INDUSTRIAIS ATÉ A INDUSTRIA 4.0}

\author{
THE INDUSTRIAL REVOLUTIONS UP TO INDUSTRY 4.0
}

\author{
Ruudi Sakurai - ruudisakurai@gmail.com \\ Jederson Donizete Zuchi - jederson.zuchi@fatectq.edu.br \\ Faculdade de Tecnologia de Taquaritinga (FATEC) - SP - Brasil
}

DOI: 10.31510/infa.v15i2.386

\begin{abstract}
RESUMO
O presente artigo tem como objetivo trazer um breve estudo de como se deu o processo de Revolução Industrial até os dias atuais com o surgimento da Indústria 4.0, destacando a sua implantação no Brasil. Foi realizado um levantamento bibliográfico de artigos acadêmicos e publicações de instituições que estudam o desenvolvimento dos modelos industriais e tecnológicos. O critério de inclusão baseou-se na data de publicação, últimos dez anos, e o idioma, todas as publicações deveriam estar escritas na língua portuguesa. Através da literatura consultada fica claro que as indústrias sempre buscaram evoluir e para isso acompanharam os grandes avanços tecnológicos. Diante de tais avanços, a sociedade vê surgir um novo modelo de indústria. A Indústria 4.0 traz em seu perfil a somatória das evoluções tecnológicas e uma nova forma de trabalho. A Literatura deixa claro que essa nova proposta de indústria é um grande avanço tecnológico e tende a ser a nova perspectiva de um mercado cada vez exigente e altamente voltado para a era digital.
\end{abstract}

Palavras-chave: Indústria 4.0. Tecnologia. Revolução Industrial.

\begin{abstract}
This article aims to bring a brief study of how the process of the Industrial Revolution to the present day with the emergence 4.0 industry, highlighting your deployment in Brazil.We conducted a bibliographic survey of scholarly articles and publications of institutions studying the development of the industrial and technological models. The inclusion criterion was based on the date of publication, the last ten years and the language. The inclusion criterion was based on the date of publication, the last ten years, and the language, all publications should be written in the Portuguese language. Through the literature consulted it is clear that the industries have always sought to evolve and that accompanied the great technological advances. Faced with such advances, society see a new model of industry. 4.0 industry brings in your profile the sum of technological developments and a new way of working. The literature makes it clear that this new proposal for industry is a major technological break through and tends tobe the new perspective of a market increasingly demanding and highly targeted towards the digital age.
\end{abstract}

Keywords: 4.0 industry. Technology. Indutrial Revolution. 


\section{INTRODUÇÃO}

O mundo assistiu ao surgimento da Indústria, um dos marcos para a evolução histórica da humanidade. Com o passar dos anos e frente as constantes inovações tecnológicas a indústria passou por importantes e profundas mudanças, todas serviram para mudar de forma considerável o desenvolvimento de uma sociedade, que hoje assiste a uma transformação tecnológica sem precedentes e precisa a todo custo adaptar-se a essa realidade.

A quarta Revolução Industrial ou a Indústria 4.0 tem seu termo usado pela primeira vez em 2011, é oriunda de um projeto de estratégias do governo alemão votado para a tecnologia (SILVEIRA, 2017).

Venturelli (2017) esclarece que a Industria 4.0 tem como foco a conectividade, ou seja, conectar toda uma indústria, desde a produção até o sistema de vendas é uma realidade dessa nova Revolução Industrial.

Como esse novo conceito de indústria surgiu na Europa e por ser ainda muito novo, sua difusão no Brasil ainda é limitada, embora seja uma estratégia a ser adotada por grandes indústrias como forma de vencer a competitividade nacional (OLIVEIRA; SIMÕES, 2017).

Segundo Silveira (2017) por ser uma nova proposta que demanda mão-de-obra qualificada e pelos altos custos para a implantação, pelo menos no Brasil, talvez a Indústria 4.0 demore para alcançar o setor industrial de forma considerável.

O objetivo principal deste estudo, fundamenta-se na importância do conhecimento a respeito da Indústria 4.0 e como o Brasil tem caminhado rumo a implantação dessa nova Revolução Industrial.

A Literatura deixa claro que a implantação e o conhecimento a respeito da Indústria 4.0 é uma necessidade para as empresas e profissionais, cujo interesse é consolidar-se no mercado das grandes e modernas indústrias. Antes de conhecer a Indústria 4.0 é importante reconhecer as revoluções industriais existentes.

\section{A EVOLUÇÃO DAS INDÚSTRIAS E A INDÚSTRIA 4.0}

Marson (2014) afirma que historicamente a indústria foi o fator mais poderoso de aceleração do crescimento econômico. O setor industrial exerceu grande impacto em diversos setores da economia e sobre todo o ambiente institucional e social. 
Os avanços tecnológicos sempre foram extremamente importantes para a evolução da Indústria, fator ocasionado pela busca de trabalhos mais dinâmicos, eficientes e qualificados. As revoluções industriais, somadas aos grandes avanços tecnológicos culminaram no surgimento da Indústria 4.0 ou quarta Revolução Industrial (BOETTCHER, 2015).

Antes de desvendar as características da Indústria 4.0 se faz importante entender um pouco do processo do surgimento das Indústrias até a Revolução Industrial que norteia os dias atuais.

\subsection{Primeira Revolução Industrial- Indústria 1.0}

Antes do surgimento da indústria, tudo era produzido de forma manual, fator que propiciava pequenas produções, e isso era inviável diante de uma população que crescia descontroladamente. Além disso, produzir mais rápido e em maior quantidade era a essência do capitalismo, que tinha como objetivo principal a obtenção de lucros (CAVALCANTE; SILVA, 2011).

A produção manual para o regime capitalista não era mais interessante. Boettcher (2015) explica que a primeira Revolução Industrial ocorreu na Inglaterra, final do século XVIII e início do século XIX, entre 1760 e 1860, e depois se estendeu para outros países como: França, Bélgica, Holanda, Rússia Alemanha e Estados Unidos.

Esse processo de Revolução Industrial ficou conhecido por importantes invenções, que provocaram a evolução do setor produtivo e de transporte. A ciência descobria a utilidade do carvão como fonte de energia e então a descoberta da máquina a vapor e a locomotiva (VENTURELLI, 2017).

De acordo com Boettcher (2015) na Industria 1.0 houve o aperfeiçoamento da máquina a vapor por James Watt. A indústria têxtil foi a primeira a utilizar a nova tecnologia da máquina a vapor. Depois disso muitos outros setores resolveram utilizar o meio de automação de processos e assim inseriram máquinas a seus processos produtivos onde a indústria têxtil passou a ser o símbolo da produção excedente.

É importante ressaltar que a primeira Revolução Industrial foi a grande motivação para o surgimento do capitalismo, que antes era comercial e passou a ser industrial. Essa revolução mudou consideravelmente a vida das pessoas e até hoje seus reflexos podem ser vistos e continuam em processo de transformação (CAVALCANTE; SILVA, 2011). 


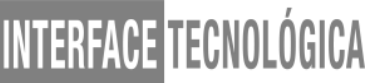

As mudanças e evoluções tecnológicas continuaram a acontecer e surge então neste contexto de inovação a Indústria 2.0.

\subsection{Segunda Revolução Industrial - Indústria 2.0}

Boettcher (2015) explica que durante o processo da Revolução das Indústrias o uso de novas tecnologias se tornou um fator essencial, para o crescimento e modernização, o que interessava os donos das indústrias interessados em aumentar cada vez mais seus lucros. Dentro deste contexto, o modelo industrial desenvolvido inicialmente sofreu mudanças importantes. Em 1870 frente a uma nova demanda tecnológica e movido pelas inovações, surge a Segunda Revolução Industrial.

A Indústria 2.0 sob o enfoque de inovações tecnológicas assumiu novas características. Nesse período foi descoberta a eletricidade, a transformação do ferro em aço, o surgimento e modernização dos meios de transporte, o avanço dos meios e comunicação, o desenvolvimento da indústria química e de outros setores. Essa revolução industrial teve destaque ela busca de maiores lucros; especialização do trabalho; ampliação da produção. (SILVA; GASPARIN, 2013).

$\mathrm{Na}$ Indústria 2.0 iniciou-se o Fordismo, termo criado por Henry Ford em 1914. O fordismo se referia aos sistemas de produção em massa. Tinha em vista racionalizar a produção capitalista por meio de inovações técnicas, onde de um lado acontecia a produção em massa e de outro o consumo em massa (BOETTCHER, 2015).

Ford criou o processo de semi-automatização o que causou uma revolução na indústria automobilística. Silva e Gasparin (2013) explicam que em 1914 Ford introduziu a primeira linha de montagem automatizada, com esteiras rolantes.

Segundo Boettcher (2015) frente a essas inovações, as indústrias alcançaram lucros cada vez maiores e qualificaram o processo desde a obtenção da matéria-prima até o consumidor final. Um aspecto bastante positivo da Indústria 2.0 era um maior controle sobre os gastos, o que consequentemente ocasionava cálculos mais precisos a respeito das margens de lucro.

Dentro deste contexto, o capitalismo tornou-se responsável pela aceleração e crescimento da economia mundial, países como Estados Unidos, Alemanha, Japão e França devido ao fato de serem países economicamente desenvolvidos acabaram tornando-se líderes globais de Tecnologia. 


\subsection{Terceira Revolução Industrial- Indústria 3.0}

Silva et. al., (2002) esclarecem que a Terceira Revolução Industrial surge como consequências dos avanços tecnológicos do século XX e XXI. Os autores explicam que mais do que um desejo tecnológico a Indústria 3.0 trouxe uma renovação no processo econômico, político e social, com grande dinamismo e alta complexidade.

Frente as grandes descobertas e inovações tecnológicas, a Terceira Revolução Industrial, também chamada de Revolução Técnico-Científica e Informacional são formadas por meio dos processos de inovação tecnológica, os quais são marcados pelos avanços no campo da informática, robótica, das telecomunicações, dos transportes, da biotecnologia, química fina, além da nanotecnologia (BOETTCHER, 2015).

Muitas foram as características da Industria 3.0 as quais configuram-se: utilização de várias fontes de energia; uso crescente de recursos da informática; aumento da consciência ambiental; diminuição crescente do desemprego, pois a mão-de-obra passou a ser substituída por maquinas cada vez mais modernas; ampliação dos direitos trabalhistas; globalização; surgimento de potencias industriais; massificação dos produtos tecnológicos (SILVA et al., 2002).

Frente a essa intensa modernização, mudança social, cultural e econômica, assim como tem feito ao longo dos anos, o homem continuou investindo em desenvolvimento tecnológico e assim nasce a Indústria 4.0.

\subsection{A Quarta Revolução Industrial - A Indústria 4.0}

Segundo Kagermann et al., (2013) o termo Indústria 4.0 surgiu publicamente em 2011 na Alemanha na feira de Hannover. Essa nova proposta de indústria surgiu por meio da necessidade de se desenvolver uma abordagem para fortalecer a competitividade da indústria manufatureira alemã.

Em 2012 os criadores do projeto ministrado por Siegfried Dais (Robert Bosch GmbH) e Kagermann (acatech) apresentou um relatório de recomendações para o Governo Federal Alemão, como forma de planejar como seria a implantação da Indústria 4.0. Em 2013 acontece na fera de Hannover a edição final sobre essa nova perspectiva industrial. (SILVEIRA, 2017). 
A Tendência desse novo modelo industrial baseia-se como a forma de descrever a tendência da digitalização e automação do ambiente de manufatura (OESTERREICH; TEUTEBERG, 2016).

Conforme Silveira (2017) o fundamento básico da Indústria 4.0 é de que conectando máquinas, sistemas e ativos, as empresas podem criar redes inteligentes e assim controlar os módulos de produção de forma autônoma.

Sobre o conceito da Indústria 4.0 Zawadzki e Zywicki (2006) esclarecem que esse novo modelo de indústria é a combinação das conquistas tecnológicas dos últimos anos com a visão de um futuro com sistemas de produção inteligentes e automatizados, no qual o mundo real é ligado a virtual.

\subsubsection{Princípios da Indústria 4.0}

Sobre os princípios da Indústria 4.0 Silveira (2017) explica que existem seis, de extrema importância e que devem ser seguidos para a implementação da quarta revolução industrial, são eles:

- Capacidade de operação em tempo real - aquisição e tratamento de dados em tempo real, fator que possibilita que decisões sejam tomadas em tempo real;

- Virtualização - essa moderna proposta industrial possui uma cópia virtual das fábricas inteligentes, permitindo assim a rastreabilidade e o monitoramento remoto;

- Descentralização - as decisões podem ser feitas pelo sistema cyber-físico, como forma de atender as necessidades de produção em tempo real;

- Orientação de Serviços - Utilização de arquiteturas de software orientadas a serviços aliado ao conceito de Internet of Services;

- Modularidade - produção de acordo com a demanda, acoplamento e desacoplamento de módulos na produção. Essa mobilidade permite alterar as tarefas das máquinas facilmente.

- Interoperabilidade - Capacidade dos sistemas cyber-físicos (suportes de peças, postos de reunião e produtos), humanos e fábricas inteligentes comunicar-se uns com os outros por intermédio da Internet das Coisas e da Internet.

Muitas são as tecnologias que podem ser usadas dentro da Indústria 4.0, no entanto, algumas são as que mais se destacam e constituem assim os pilares da quarta Revolução Industrial. 


\subsubsection{Pilares da Indústria 4.0}

As tecnologias mais relevantes para a implementação e funcionamento da Indústria 4.0 podem ser assim definidas:

- Internet das Coisas: “a relação entre coisas (produtos, serviços, lugares) e pessoas, por meio de plataformas e tecnologias conectadas" (SCHWAB, 2016, p. 26). Silveira (2017) explica que os sistemas que funcionam a base da Internet das Coisas, são dotados de sensores e atuadores e são denominados de sistemas Cyber-físicos, e são a base da indústria;

- Segurança cibernética: "meios de comunicação cada vez mais confiáveis e sofisticados" (RUBMANN et al., 2015, p. 6). O maior sucesso de um programa ou produto altamente tecnológico é a segurança. Os problemas ou falhas podem comprometer todo um trabalho a ser desenvolvido (SILVEIRA, 2017);

- Big Data Analytics: são estruturas de dados extensas e complexas que utilizam novas abordagens para captura, análise e gerenciamento de informações. Aplicada à indústria 4.0, a tecnologia de Big Data é estruturada em 6Cs como forma de lidar com as informações mais relevantes e importantes: Conexão (à rede industrial, sensores e CLPs), Cloud (nuvem/dados por demanda), Cyber (modelo e memória), Conteúdo, Comunidade (compartilhamento das informações) e Customização (personalização e valores) (SILVEIRA, 2017);

- Computação em nuvem: "banco de dados capaz de ser acessado de qualquer lugar do mundo em milissegundos, por meio de dispositivos conectados à internet" (RUBMANN et al., 2015, p. 6-7);

- Robótica avançada: "robôs mais adaptáveis e flexíveis; futuramente, sua interação com outras máquinas e humanos será uma realidade cotidiana" (SCHWAB, 2016, p. $25)$;

- Inteligência artificial: "pode reduzir custos, proporcionar ganhos de eficiência e até mesmo “computadorizar" empregos" (SCHWAB, 2016, p. 141-142);

- Novos materiais: "são mais leves e fortes, recicláveis e adaptáveis; podem ser "inteligentes" com propriedades como autorreparação ou autolimpeza" (SCHWAB, 2016, p. 25).

Diante de tanta inovação e tecnologia é preciso se preparar para inserir em um mercado uma Indústria com tantas perspectivas de crescimento. Com relação aos avanços 
tecnológicos o Brasil tem apresentado pontos positivos. Realmente a Indústria 4.0 passa a fazer parte de uma realidade brasileira não tão distante.

\subsection{Os Impactos da Indústria 4.0}

O maior de todos os impactos produzidos pela Indústria 4.0 é a mudança que a mesma irá trazer em todo o contexto. Essas mudanças consistem por exemplo, em novos modelos de negócio e um mercado cada vez mais exigente. Devido ao fator de rapidez da automação os produtos podem ser customizados, e esse fator tende a ser uma variável a mais no processo de manufatura, mas as fábricas inteligentes serão capazes de levar a personalização de cada cliente em consideração, se adaptando às preferências (SILVEIRA, 2017).

Ainda segundo Silveira (2017) um setor que tende a sofrer o impacto da Indústria 4.0 será o departamento dos campos de pesquisa e desenvolvimento de segurança da Tecnologia da Informação (TI), o setor de confiabilidade do produto e a interação máquina-máquina.

Como forma de conseguir atender essa nova demanda e modelo de indústria a tecnologia deve sofrer um processo de evolução contínua. É preciso também que os profissionais adequem sua capacidade de trabalho a essa nova realidade de grandes avanços tecnológicos. Capacitar esses empregados é uma das estratégias para atender as indústrias oriundas dessa nova Revolução Industrial

\subsection{A Indústria 4.0 no Brasil}

Segundo Hahn (2017) no Brasil com relação aos avanços tecnológicos a indústria nacional se encontra em atraso, e isso torna-se ainda mais evidente e concreto quando compara-se a indústria brasileira com países como a Alemanha, Estados Unidos e Japão. A substituição de linhas tradicionais com pessoas por linhas de automação ainda apresenta um ritmo muito inferior aos países desenvolvidos.

A Confederação Nacional da Indústria (CNI) (2016) a indústria brasileira embora busque se inserir a indústria e tem como objetivo inicial o desenvolvimento de melhores produtos e modelos de negócio.

Zancul (2016) afirma que muitas indústrias brasileiras já se automatizaram, no entanto, a manufatura digital ainda não foi alcançada. $\mathrm{O}$ autor esclarece que a indústria 4.0 é formada por duas vertentes: processos integrados e produtos inovadores, e o Brasil em relação 
a essas vertentes precisa caminhar muito, uma vez que na escala global o país tem poucos setores competitivos.

Muitas empresas brasileiras ainda estão entrando na Terceira Revolução Industrial. A Indústria no Brasil segundo o autor se encontra em diferentes níveis de desenvolvimento tecnológico, no entanto é possível pular etapas e acompanhar os países inseridos na quarta Revolução Industrial. As pequenas e médias empresas podem investir nessas novas tecnologias e assim obter maiores ganhos e aumentar o poder de competitividade (SANTOS, 2017).

No Brasil o conceito de Indústria 4.0 pode ser introduzido e adequado de acordo com a realidade nacional, ao qual, deve-se pular etapas e aproveitar todos os conceitos de melhoria que essa Revolução Industrial propõe. A dificuldade de implantação no país acontece devido a falta de conhecimento sobre o processo ou resistência pela novidade desse projeto (HAHN, 2017).

\section{PROCEDIMENTOS METODOLÓGICOS}

A metodologia utilizada para o desenvolvimento deste artigo foi pesquisa bibliográfica, onde foram consultados livros, artigos, teses, monografias, revistas, documentos apresentados em simpósios e congressos e sites da internet. Todos os documentos tinham como foco os assuntos que estruturam este estudo.

\section{RESULTADOS E DISCUSSÃO}

Segundo a CNI (2016) o maior problema para que a Indústria 4.0 seja implantada no Brasil está no conhecimento ainda muito abaixo do que se deve esperar. Os dados obtidos através das pesquisas apontam que $48 \%$ das indústrias brasileiras utilizam ao menos uma tecnologia, se comparado as grandes empresas esse valor sobe para $63 \%$ e em relação as pequenas empresas cai para $25 \%$.

Mesmo diante as dificuldades de implantação Boucherat (2016) explica que algumas empresas estão procurando iniciar o processo de automação com grande evolução tecnológica, e o início nesse processo tem apresentado resultados positivos. O autor cita a Volkswagen do Brasil, a qual desde 2008 utiliza os conceitos da indústria 4.0 e percebeu 


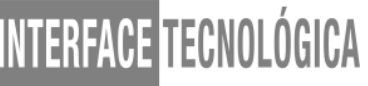

melhorias na eficiência e no sistema produtivo. O autor esclarece que outra empresa que tem investido tecnologicamente em seu processo produtivo é a Bosch Rextoth.

A Bosch obteve aumento de $25 \%$ em sua produção de Sistema de Frenagem Automática (ABS) e do Programa de Estabilidade Eletrônica (EPS), tal melhoria foi resultado da implantação de linhas inteligentes conectadas. A implantação dos conceitos da Indústria 4.0 tende a trazer benefícios financeiros além da otimização dos recursos (BOUCHERAT, 2016).

De acordo com Silveira (2017) o uso da alta tecnologia diminui as chances do erro humano, reduz os custos, aumenta a capacidade produtiva e eficiência em todos em todos os processos.

A CNI (2016) através de uma pesquisa realizada, destaca que para acelerar a implantação dessas tecnologias no Brasil o governo deverá investir em infraestrutura digital, promover linhas de financiamento para o mercado e incentivar a educação através de treinamentos para que o conhecimento seja levado aos profissionais nesse novo cenário de trabalho.

As empresas em transição digital precisarão de investimentos consideráveis além de mudanças significativas $(\mathrm{KOCH}, 2014)$. Embora o Brasil esteja caminhado rumo ao desenvolvimento tecnológico e apresente aspectos positivos nesse sentido, ainda é preciso investir muito e buscar adequação a essas novas transformações que a Indústria 4.0 proporciona.

\section{CONSIDERAÇÕES FINAIS}

O mundo assistiu alguns processos de Revolução Industrial, todas elas com o objetivo de alavancar o processo produtivo e adequar o mesmo as tecnologias existentes em cada época.

A Industria 4.0 surge mediante a um cenário de grandes evoluções tecnológicas e traz em seu contexto projetos de uma indústria do futuro. Embora seja um projeto com aspectos bastante positivos seus impactos são notáveis e tendem a mudar profundamente a sociedade.

O Brasil tem se inserido no processo da globalização e embora produto de uma industrialização e avanço tecnológico tardio, tem mostrado motivação e vontade de crescer. 


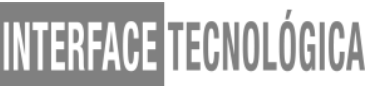

\section{REFERÊNCIAS}

BOETTCHER, M. Revolução Industrial - Um pouco de história da Indústria 1.0 até a Indústria 4.0. Linkedin. 26 nov. 2015. Disponível em:< https://pt.linkedin.com/pulse/revolu\%C3\%A7\%C3\%A3o-industrial-um-pouco-dehist\%C3\%B3ria-da-10-at\%C3\%A9-boettcher>. Acesso em: 10 maio 2108.

BOUCHERAT, X. Industry 4.0 and the rise of smart manufacturing. Automotive Megatrends Magazine, Q2, p. 59-61, 2016.

CAVALCANTE, Z. V.; SILVA, M. L. S. da. A importância da Revolução Industrial no mundo da Tecnologia. In: ENCONTRO INTERNACIONAL DE PRODUÇÃO CIENTÍFICA, 7. 2011. Maringá. Anais eletrônico. Maringá. 2011. Disponível em:< https://www.unicesumar.edu.br/epcc-2011/wp-

content/uploads/sites/86/2016/07/zedequias_vieira_cavalcante2.pdf $>$. Acesso em: 12 jun. 2018.

CEMBRANELLI, F. O que é a 4 Revolução Industrial e qual seu impacto na saúde. Health Hinnova Hub. 19. jan. 2018. Disponível em:< https://startupsaude.com/o-que-e-a-4revolucao-industrial-e-qual-seu-impacto-na-saude/>. Acesso em: 12 jul. 2018.

CONFEDERAÇÃO NACIONAL DA INDÚSTRIA (CNI). Desafios para Indústria 4.0 no Brasil. Brasília: CNI, 2016.

KAGERMANN, $\mathrm{H}$ et al. Recommendations for implementing the strategic iniative Industrie. 2013.

KOCH. V. Industry 4.0: Opportunities and challenges of the industrial internet. PWC. 2016.

OESTERREICH, T. D.; TEUTEBERG, F. Understanding the implications of digitisation and automation in the context of Industry 4.0: A triangulation approach and elements of a research agenda for the construction industry. Computers in Industry. 2016.

OLIVEIRA, F. T. de; SIMÕES, W. L. A Indústria 4.0 e a produção no contexto dos Estudantes de Engenharia. In: SIMPÓSIO DE ENGENHARIA DE PRODUÇÃO. 2017. Goiás. Anais eletrônicos. Goiás, 2017. Disponível em:< https://sienpro.catalao.ufg.br/up/1012/o/Fernanda_Tha\%C3\%ADs_de_Oliveira.pdf >. Acesso em: 20 de jun. 2018.

MARSON, M. D. A industrialização brasileira antes de 1930: uma contribuição sobre a evolução da indústria de máquinas e equipamentos no estado de São Paulo, 1900-1920. SciELO, São Paulo, out/dez. 2014. Disponível em:< http://www.scielo.br/scielo.php?script=sci_arttext\&pid=S0101-41612015000400753>. Acesso em: 12 jul. 2018. 
RUBMANN, M.; LORENZ, M.; GERBERT, P.; WALDNER, M.; JUSTUS, J.; ENGEL, P.; HARNISCH, M. Industry 4.0: The Future of Productivity and Growth in Manufacturing Industries. The Boston Consulting Group: BCG, 2015.

SCHWAB, K. A quarta revolução industrial. São Paulo: Edipro, 2016.

SILVA, M. C. A. da.; GASPARIN, J. L. A Segunda Revolução Industrial e suas influências sobre a Educação Escolar Brasileira. 2015. Disponível em:< http://www.histedbr.fe.unicamp.br/acer_histedbr/seminario/seminario7/TRABALHOS/M/Ma rcia\%20CA\%20Silva\%20e\%20\%20Joao\%20L\%20Gasparin2.pdf>. Acesso em: 20 jun. 2018.

SILVA, D. B. da. et al. O Reflexo da Terceira Revolução Industrial na Sociedade. In: ENCONTRO NACIONAL DE ENGENHARIA DE PRODUÇÃO, 22., 2012, Curitiba. Curitiba, ABEPRO, 2012. Disponível em:

http://www.abepro.org.br/biblioteca/enegep2002_tr82_0267.pdf. Acesso em: 20 jun. 2108.

SILVEIRA, C. B. O que é a Indústria 4.0 e como ela vai impactar o mundo. Citisystems. 2017. Disponível em: $<$ https://www.citisystems.com.br/industria-4-0/>. Acesso em: 10 jun. 2018.

VENTURELLI, M. Indústria 4.0: uma visão da automação industrial. Automação Industrial, nov. 2017. Disponível em:< https://www.automacaoindustrial.info/industria-4-0uma-visao-da-automacao-industrial/>. Acesso em: 10 jul. 2108.

ZANCUL, E. de S. O Brasil está pronto para a Indústria 4.0? Exame. São Paulo, maio. 2016. Disponível em: $<$ https://exame.abril.com.br/tecnologia/o-brasil-esta-pronto-para-a-industria4-0/>. Acesso em: 30 jul. 2018.

ZAWADZKI, P.; ŻYWICKI, K. Smart product design and production control for effective mass customization in the Industry 4.0 concept. Management and Production Engineering Review. 2016. 